\title{
Neurotoxic or Neuroprotective? Current Controversies in SCI-Induced Autoimmunity
}

\author{
Jonah W. Saltzman • Ricardo A. Battaglino • \\ Helen L. Stott • Leslie R. Morse
}

Published online: 28 June 2013

(c) Springer Science + Business Media New York 2013

\begin{abstract}
Controversy exists regarding the autoimmune response that has been observed following traumatic spinal cord injury (SCI). It is not clear if this represents a protective response by the immune system to prevent further tissue damage, a pathological reaction of the immune system to central nervous system antigens released by the injury, or a combination of both. Experimental evidence indicates that B cells produce auto-antibodies following SCI and that the presence of self-reactive antibodies is associated with tissue damage. Conversely, other studies suggest $\mathrm{T}$ cell activity at the site of the injury promotes tissue regeneration. Vaccination with dendritic cells exposed to central nervous system antigens dramatically improves recovery of motor function in spinal cord injured rats. Further research is required to determine the nature of post-SCI B cell and T cell responses and to establish efficacy of dendritic cell vaccination therapy in clinical studies. This information is critical for the development of therapies to either suppress or promote immune responses following neurotrauma to improve neurological outcomes.
\end{abstract}

J. W. Saltzman · R. A. Battaglino · L. R. Morse

Department of Mineralized Tissue Biology, Forsyth Institute,

Boston, MA, USA

R. A. Battaglino

Department of Oral Medicine, Infection, and Immunity, Harvard

School of Dental Medicine, Boston, MA, USA

H. L. Stott · L. R. Morse ( $\square)$

Spaulding-Harvard Spinal Cord Injury Model System, Department of PMR, Spaulding Rehabilitation Hospital, Harvard Medical School, 125 Nashua Street, Boston, MA 02114, USA

e-mail: 1morse4@partners.org

L. R. Morse

Department of Physical Medicine and Rehabilitation, Harvard

Medical School, Boston, MA, USA
Keywords Spinal cord injury $\cdot \mathrm{B}$ cell $\cdot \mathrm{T}$ cell $\cdot$ Dendritic cell · Autoimmunity $\cdot$ Rehabilitation

\section{Introduction}

Traumatic spinal cord injury (SCI) has been observed to provoke a systemic inflammatory response in the chronic phase of injury that can last for decades [1]. There is evidence that this response is autoimmune in nature [2-6], a phenomenon that has been reviewed at length [5, 7]. However, the question of whether this SCI-induced autoimmune response is pathological, neuroprotective, or a combination of both remains unresolved and is the subject of some controversy. The answer to this question would significantly impact research into the mitigation of neuroinflammation in individuals with SCI, indicating either that the observed autoimmunity should be alternatively suppressed or modulated to improve outcomes [8]. This review will focus on recent work demonstrating neuroprotective or neurotoxic effects of SCI-induced autoimmunity.

\section{B Cell Mediated Pathological Autoimmunity}

There is much evidence that $\mathrm{B}$ cells mediate a harmful autoimmune response following SCI. Ankey et al. [4] have shown that B cell knockout mice experienced a smaller reduction in motor function after SCI than wild-type mice. Elevated autoantibody titers in the sera of SCI subjects were shown to correlate with secondary consequences of autoimmunity including renal dysfunction, sterility, neuropathic pain, and pressure ulcers [9]. More recently, mice lacking B cells and subjected to compression SCI, demonstrated improved recovery compared to WT mice, as evidenced by greater reinnervation of the spinal cord, improved 
locomotive function, additional regrowth of axons at the injury site, and preservation of myelin sheaths in the ventral column $\left[10^{\bullet} \cdot\right.$. The production of auto-antibodies is thought to cause pathology by inducing demyelination, directing the complement system against CNS tissue, and recruiting cytotoxic $\mathrm{T}$ cells into the CNS [11].

The $\mathrm{B}$ cells are thought to promote autoimmune responses through the secretion of proinflammatory cytokines [12] and the production of self-reactive antibodies [13]. In 2006, Ankey et al. [1] observed the presence of antibodies reactive with self CNS proteins in the sera of spinal cord injured mice, and demonstrated that SCI elicits the production of antiDNA and anti-RNA antibodies. Production of anti-nucleic acid auto-antibodies in Lupus-prone mice requires stimulation of nucleic acid-sensing toll-like receptors (NA-TLRs) on the surface of B cells. Furthermore, NA-TLRs are required for the production of the anti-chromatin and rheumatoid factor antibodies [14]. Though never demonstrated, it is possible that stimulation of B cell NA-TLRs is required for the production of anti-nucleic acid auto-antibodies after SCI.

Alterations in cytokine levels may play a role in immunomodulation after SCI. Two cytokines, a proliferation inducing ligand (APRIL) and $\mathrm{B}$ cell activating factor (BAFF), are implicated in the maintenance of autoimmune responses by promoting the survival of self-reactive B cells (B cells producing auto-antibodies) [15-17]. We recently observed that $\mathrm{B}$ cell progenitors from individuals with chronic SCI over-express both of these factors compared to individuals without SCI, and speculate that this overexpression promotes self-reactive B cell development in SCI [18••]. Interleukin-10 (IL-10) is an anti-inflammatory cytokine with a wide array of functions in the immune system. In addition to suppressing effector $\mathrm{T}$ cell activity, IL-10 has been observed to enhance B cell activity, including antibody synthesis and costimulatory molecule production with seemingly contrary effects $[19,20]$. In 2013, Paulson et al. [21•] reported increased plasma concentrations of IL-10 in human subjects with SCI. Owing to the diverse effects of IL10 on the various cells of the immune system, it is unknown if increased IL-10 promotes or suppresses SCI-induced autoimmunity, and if it is the former, whether this effect is beneficial or not.

\section{Dendritic Cell and T Cell Mediated Protective Autoimmunity}

The concept of protective autoimmunity has recently become the subject of intensive investigation in the context of neurological trauma. Recent evidence suggests that the trauma-elicited autoimmune response can be neuroprotective by preventing tissue damage and stimulating tissue repair [22-24]. This protective autoimmunity is mediated, in part, by autoreactive $\mathrm{T}$ cells, which play a role in recruiting other resident CNS cells implicated in tissue repair and regeneration $[22,25]$. The window for the induction of a protective or reparative autoimmune response is relatively small, making immune-based treatments in the acute phase of the injury difficult [25]; however, the development of an autoimmune intervention during the acute phase of SCI could mitigate or prevent further neurological damage. In fact, Schwartz et al. [22] suggest that autoimmunity is a natural response to CNS damage, and that related pathology and neurological damage is the result of an inability to properly control and direct the autoimmune reaction. Thus, any therapy that could assist in the control or targeting of a post-SCI autoimmune response could support native reparative mechanisms. Schwartz et al., indicate that the same T cells are responsible for both neuroprotective and neurodegenerative effects, and show that stimulation of $\mathrm{T}$ cell activity can be achieved without significant risk. Therefore, therapies involving induced autoimmunity may be beneficial.

Dendritic cells (DCs) are professional antigen-presenting cells (APCs) that are the primary presenters of antigen to $T$ cells. Over the last decade, DCs have emerged as the focus of much research at the intersection of CNS trauma and autoimmunity, particularly for their status as the most frequent and most potent activators of naïve $\mathrm{T}$ cells and for their potential to ameliorate various forms of neurological damage after SCI. A commonly used method of studying the role of dendritic cells in autoimmune pathologies is DC vaccination. In this method, dendritic cells are exposed to a particular antigen or antigen cocktail in vitro, and are then injected into injured animals. Dendritic cells are extremely efficient at taking up this antigen and presenting it on the cell surface coupled to MHC class II molecules. Antigen presentation also causes DCs to express surface antigens that allow them to activate $\mathrm{T}$ cells that recognize the MHC II: antigen complex on the surface of the dendritic cell. Injection of dendritic cells following SCI in rodent models has been shown to activate neural stem/progenitor cells causing neurogenesis and neurological recovery [26], to protect from neurological damage and promote neurological recovery (after having been treated with CNS antigens) [27••], and to cause early locomotive recovery in the presence of granulocyte macrophage colony-stimulating factor (GM-CSF) [28]. However, there is evidence that the function and activity of DCs is suppressed after SCI. The DCs extracted from subjects with SCI are shown to express fewer markers of activation in response to stimulation with LPS, and demonstrate an impaired ability to cause the activation and proliferation of naïve T cells [29]. Significantly, however, the same study showed no difference due to SCI in the reactivity and lymphoproliferative response (LPR) of T cells in response to LPS. Recent evidence in mice has shown that injection of dendritic cells that have 
been exposed to spinal cord homogenate $(\mathrm{SCH})$ ex vivo has a neuroprotective effect, accompanied by migration of CD4+ T cells to the SCI lesion and an increase in the levels of circulating proinflammatory cytokines [27••]. This further illustrates the protective role that autoimmune $\mathrm{T}$ cells can have in the context of SCI, and, importantly, suggests that beneficial autoimmunity after SCI specifically targets antigens found in the CNS.

\section{T Cell Mediated Pathological Autoimmunity}

Despite the many studies reporting positive effects of various DC and T cell-based treatments, there is still much controversy regarding the role of T cells in SCI-induced autoimmunity. As recently as 2012, one group reported that the transfer of the Th1 subset of the $\mathrm{T}$ cell population to spinal cord injured mice improved post-SCI recovery, while also noting that the transfer promoted the activity of certain microglia/macrophage subtypes that resulted in significant neutralization of circulating IL-10, promoting inflammation and reducing the benefits of the cell transfer [30॰]. Jones et al. [31] performed an experiment similar to Wang et al. using myelin basic protein instead of spinal cord homogenate, and reported that the immunization impaired neurological function and worsened neuropathology after SCI in rats. In another study, the presence of myelin basic protein-reactive $\mathrm{T}$ cells in entorhinal-hippocampal slice cultures resulted in microglia-mediated demyelination and axon loss to a greater extent than that of autoreactive $\mathrm{T}$ cells specific to non-CNS antigens, indicating that the specificity of the T cells to MBP was responsible for the observed neuropathology [32]. Another group reported that the transfer of activated CD4+ $\mathrm{T}$ cells to rats pre-injury severely exacerbated the effects of an aseptic cerebral injury, and that administration of $\mathrm{T}$ cell suppressing agents (cyclosporin A and FK506) attenuated the effects of the injury [33]. Similarly, the same study that demonstrated the benefits of B cell knockout reported similar effects of $\mathrm{T}$ cell knockout in mice [10••]. A recent study showed that the incomplete maturation of Th1 and Th17 helper $T$ cells was associated with resistance to experimental autoimmune encephalomyelitis (EAE) [34], indicating that $T$ cells may play a pathological role in autoimmune processes.

\section{Further Areas of Investigation}

The presence of contradictory results in the literature necessitates ongoing work to better define the SCI-induced autoimmune reaction. $\mathrm{T}$ cells and $\mathrm{B}$ cells alike have been shown to attenuate or exacerbate the effects of CNS trauma and autoimmune responses. Defining key biological pathways leading to protective or pathological immune responses will lead to identification of novel therapeutic targets. Multiple studies in rodent models have demonstrated the benefits of DC-based therapies following stroke, $\mathrm{SCI}$, traumatic brain injury, and experimental lupus. The known neuroprotective effects of dendritic cells, coupled with the observation that DCs experience suppression in $\mathrm{SCI}$, present an inviting target for future therapies in chronic SCI and other CNS trauma. Future work is required to determine if DC vaccination therapy is effective in humans. The question of whether NA-TLR stimulation is required for anti-nucleic acid autoantibody production in chronic SCI should be explored and therapies designed to prevent NA-TLR stimulation (for example, neutralization of circulating NA-TLR ligands using recombinant antibodies) could be studied in the context of neurologic recovery. Further defining the role of cytokines following SCI and possibly targeting circulating BAFF and APRIL in an effort to improve neurologic outcome after injury could also be considered. In conclusion, exploring the role of auto-immunity as it pertains to preventing neurologic damage or facilitating neurologic recovery following SCI is a vast, exciting and promising area of research that deserves further and sustained efforts.

Acknowledgments This study received support from: the National Institute of Child Health and Human Development [R21HD057030 and R21HD057030-02S1], the National Institute of Arthritis and Musculoskeletal and Skin Diseases [1R01AR059270-02], and the Department of Education, NIDRR H133N110010.

Conflict of Interest J.W. Satzman, R.A. Battaglino, H. Stott, L.R. Morse declare they have no conflicts of interest.

Human and Animal Rights and Informed Consent This article does not contain any studies with human or animal subjects performed by any of the authors.

\section{References}

Papers of particular interest, published recently, have been highlighted as:

- Of importance,

•- Of major importance

1. Ankeny DP, et al. Spinal cord injury triggers systemic autoimmunity: evidence for chronic B lymphocyte activation and lupuslike autoantibody synthesis. J Neurochem. 2006;99(4):1073-87.

2. Hayes KC, et al. Elevated serum titers of proinflammatory cytokines and CNS autoantibodies in patients with chronic spinal cord injury. J Neurotrauma. 2002;19(6):753-61.

3. Kil K, et al. $\mathrm{T}$ cell responses to myelin basic protein in patients with spinal cord injury and multiple sclerosis. J Neuroimmunol. 1999;98(2):201-7.

4. Ankeny DP, Guan Z, Popovich PG. B cells produce pathogenic antibodies and impair recovery after spinal cord injury in mice. J Clin Invest. 2009;119(10):2990-9. 
5. Ankeny DP, Popovich PG. B cells and autoantibodies: complex roles in CNS injury. Trends Immunol. 2010;31(9):332-8.

6. Dekaban GA, Thawer S. Pathogenic antibodies are active participants in spinal cord injury. J Clin Invest. 2009;119(10):2881-4.

7. Ankeny DP, Popovich PG. Mechanisms and implications of adaptive immune responses after traumatic spinal cord injury. Neuroscience. 2009;158(3):1112-21.

8. Popovich PG, Longbrake EE. Can the immune system be harnessed to repair the CNS? Nat Rev Neurosci. 2008;9(6):481-93.

9. Davies AL, Hayes KC, Dekaban GA. Clinical correlates of elevated serum concentrations of cytokines and autoantibodies in patients with spinal cord injury. Arch Phys Med Rehabil. 2007; 88(11):1384-93.

10. $\bullet \mathrm{Wu} \mathrm{B}$, et al. Improved regeneration after spinal cord injury in mice lacking functional T- and B-lymphocytes. Exp Neurol, 2012;237(2):274-85. This study examined the effect of the deletion of recombination activating gene 2, causing an absence of mature $T$ and $B$ lymphocytes, on the outcomes of experimental SCI in mice. Mice lacking mature lymphocytes demonstrated greater improvement than the wild-type, as demonstrated by increased reinnervation of the spinal cord, better locomotive function, and greater remyelination near the injury six weeks post-SCI, indicating a pathological role of $T$ and $B$ cells in the recovery process.

11. Archelos JJ, Hartung HP. Pathogenetic role of autoantibodies in neurological diseases. Trends Neurosci. 2000;23(7):317-27.

12. Fillatreau $\mathrm{S}$, et al. B cells regulate autoimmunity by provision of IL-10. Nat Immunol. 2002;3(10):944-50.

13. Archelos JJ, Storch MK, Hartung HP. The role of B cells and autoantibodies in multiple sclerosis. Ann Neurol. 2000;47(6): 694-706.

14. Koh YT, et al. Role of nucleic acid-sensing TLRs in diverse autoantibody specificities and anti-nuclear antibody-producing B cells. J Immunol. 2013;190(10):4982-90.

15. Mackay F, Silveira PA, Brink R. B cells and the BAFF/APRIL axis: fast-forward on autoimmunity and signaling. Curr Opin Immunol. 2007;19(3):327-36.

16. Cancro MP, D'Cruz DP, Khamashta MA. The role of B lymphocyte stimulator (BLyS) in systemic lupus erythematosus. J Clin Invest. 2009;119(5):1066-73.

17. Craxton A, et al. BAFF regulates B cell survival by downregulating the BH3-only family member Bim via the ERK pathway. J Exp Med. 2005;202(10):1363-74.

18. • Saltzman JW, et al. B-cell maturation antigen, a proliferationinducing ligand, and B-cell activating factor are candidate mediators of spinal cord injury-induced autoimmunity. J Neurotrauma. 2013;30(6):434-40. In this study of 13 subjects with SCI and 7 controls, the authors found, using microarray expression and quantitative PCR analysis, that human peripheral blood mononuclear cells express higher quantities of $B$ cell survival and activation factors. Considering that $B$ cells have been implicated in post-SCI pathologies, these results indicate that overexpression of the genes examined, BAFF and APRIL, may mediate the autoimmune $B$ cell reaction observed in chronic $S C I$.

19. Moore KW, et al. Interleukin-10 and the interleukin-10 receptor. Annu Rev Immunol. 2001;19:683-765.

20. Mann MK, et al. Pathogenic and regulatory roles for B cells in experimental autoimmune encephalomyelitis. Autoimmunity. 2012;45(5):388-99.

21. Paulson TA, et al. Spinal cord injury level and the circulating cytokine response to strenuous exercise. Med Sci Sports Exerc.
2013. This is the first study to report elevated levels of circulating IL-10 in human SCI subjects in the chronic phase of the injury. Given the vital importance of IL-10 in a wide variety of immune system processes, these results call attention to a potentially very promising area of inquiry in SCI-induced autoimmunity research.

22. Schwartz M, Kipnis J. Protective autoimmunity and neuroprotection in inflammatory and noninflammatory neurodegenerative diseases. J Neurol Sci. 2005;233(1-2):163-6.

23. Yoles E, et al. Protective autoimmunity is a physiological response to CNS trauma. J Neurosci. 2001;21(11):3740-8.

24. Schwartz M, Kipnis J. Protective autoimmunity: regulation and prospects for vaccination after brain and spinal cord injuries. Trends Mol Med. 2001;7(6):252-8.

25. Ziv Y, et al. A novel immune-based therapy for stroke induces neuroprotection and supports neurogenesis. Stroke. 2007;38(2 Suppl):774-82.

26. Mikami Y, et al. Implantation of dendritic cells in injured adult spinal cord results in activation of endogenous neural stem/progenitor cells leading to de novo neurogenesis and functional recovery. J Neurosci Res. 2004;76(4):453-65.

27. •- Wang Y, et al. Neuroprotective effect of vaccination with autoantigen-pulsed dendritic cells after spinal cord injury. J Surg Res. 2012;176(1):281-92. In this study, the authors report that mice injected with DCs that have been exposed to an autologous spinal cord homogenate demonstrated greatly improved recovery from experimental SCI than mice injected with unexposed DCs. The improved recovery also correlated with infiltration of $T$ cells to the lesion site. These results strongly indicate that when DCs present CNS antigen to T cells, T cells are then able to mediate a protective autoimmune response that could play a major role in future post-SCI therapies.

28. Hayashi $\mathrm{K}$, et al. Activation of dendritic-like cells and neural stem/progenitor cells in injured spinal cord by GM-CSF. Neurosci Res. 2009;64(1):96-103.

29. Pan SC, et al. In vitro maturation potential of monocyte-derived dendritic cells is impaired in patients with spinal cord injury: a case-control study. Arch Phys Med Rehabil. 2005;86(5):974-8.

30. - Ishii H, et al. Adoptive transfer of Th1-conditioned lymphocytes promotes axonal remodeling and functional recovery after spinal cord injury. Cell Death Dis. 2012;3:e363. In this study, the authors report that intraperitoneal adoptive transfer of type 1 helper $T$ cells in mice results in recovery of locomotor activity and tactile sensation. Considering that this is the subset of the T cell population that is activated by antigen-presenting DCs, these results indicate that the interaction of T cells and DCs is part of a protective autoimmune response that promotes neurological recovery following SCI.

31. Jones TB, et al. Passive or active immunization with myelin basic protein impairs neurological function and exacerbates neuropathology after spinal cord injury in rats. J Neurosci. 2004;24(15): 3752-61.

32. Gimsa U, et al. Axonal damage induced by invading $\mathrm{T}$ cells in organotypic central nervous system tissue in vitro: involvement of microglial cells. Brain Pathol. 2000;10(3):365-77.

33. Fee D, et al. Activated/effector CD4+ T cells exacerbate acute damage in the central nervous system following traumatic injury. J Neuroimmunol. 2003;136(1-2):54-66.

34. Hertzenberg D, et al. Developmental maturation of innate immune cell function correlates with susceptibility to central nervous system autoimmunity. Eur J Immunol. 2013. 